\title{
Auf der Suche nach den chinesischen Charakteristika
}

\section{Westliche Therapieansätze in der Volksrepublik China}

\author{
Annette Hillers-Chen \\ Psychotherapie-Wissenschaft 8 (2) 11-18 2018 \\ www.psychotherapie-wissenschaft.info \\ CC BY-NC-ND \\ https://doi.org/10.30820/8243.01
}

«Die chinesische Kultur nimmt in ihren Traditionen stärker erfahrungsbasierte und subjektive Ausrichtungen an als analytische und objektive.»

Xu \& Tracey, Arizona State University, 2016, S. 359, Übers. d.A.

«Was Beratungsergebnisse betrifft, schütten westliche Menschen ihr Herz aus, östliche Menschen sind problemlöseorientiert.»

Yan, Nanjing Normal University, 2008, S. 63, Übers. d. A.

\begin{abstract}
Zusammenfassung: Psychotherapie nach «westlichem〉 Muster ist in China zu einem florierenden Markt geworden. Beim derzeitigen Stand von Psychotherapie in der Volksrepublik ist es kaum möglich zu entscheiden, welche Ansätze sich durchgesetzt hätten oder besser bzw. schlechter zu China passten, zumal die allgemeinen Erörterungsversuche basierend auf abstrakten Dichotomien und den drei Klassikern Konfuzianismus, Taoismus und Buddhismus das heutige komplexe, veränderungsschnelle Gebilde genannt China immer schlechter abbilden können. Eine Verortung «chinesischer Charakteristika> kann leicht ad absurdum geführt werden. Ein Ausweg besteht darin, die Komplexitäten und das konkrete Handeln im Feld wieder mehr in den Vordergrund zu rücken und den reziproken Prozess des Sich-miteinanderaneinander-Reibens an sich nicht als Kulturierungsversuch einer Therapieform an China zu verstehen, sondern als beidseitigen Veränderungsprozess, das heisst Kulturierung und Rekulturierung einer Therapieform als Ganzes. Die Frage nach den chinesischen Charakteristika wird dabei nebensächlich.
\end{abstract}

Schlüsselwörter: Psychotherapie, chinesische Charakteristika, Kulturierung, Indigenisierung, Volksrepublik China

Wie werden westliche Therapieformen in China aufgenommen und adaptiert? Mit dieser Fragestellung bat mich der Herausgeber dieses Hefts, einen Artikel zu diesem Themenband beizutragen. Eigentlich hatte ich angenommen, es würde mir leicht fallen, mich in dieses Thema einzuarbeiten - über 14 Jahre bin ich nun in der Volksrepublik China in den Bereichen Kulturvergleichende Psychologie und Psychotherapeutische Beratung an einer Universität tätig. Doch das moderne China mit seinen Extremen und seiner Schnelligkeit stellte mich bei diesem Thema vor einen immer grösser werdenden Berg schier unlösbarer Fragen. Am Ende hatte ich Ideen für ein ganzes Buch oder wollte einfach gar nichts mehr sagen. Was ich im Folgenden präsentieren werde, ist daher meine sehr persönliche Auswahl basierend auf meinen zugegebenermassen begrenzten Annahmen darüber, was für ein Schweizer Fachpublikum von Interesse sein könnte.

\section{Psychotherapie in China heute}

Der heutige Therapiemarkt der Volksrepublik China ist so heiss gelaufen wie kein anderer. Überall werden Wei- terbildungsangebote für Psychotherapie durchgeführt und Psychotherapiefirmen und Internetangebote spriessen aus dem Boden. Therapeutin ${ }^{1}$ oder Berater ist ein gefragtes Berufsbild geworden, obwohl es eigentlich bisher wenig Aufstiegsmöglichkeiten und kaum eine professionelle Organisation jenseits medizinischer Institutionen gibt (Zhao, 2014). Und bei ungefähr 170 Millionen Personen², die an einer DSM-klassifizierbaren psychischen Störung leiden (Phillips et al., 2009) sowie einer derzeitigen Behandlungsrate von lediglich 5\% meist in Spitälern ist die Decke dieser Entwicklung sicher noch nicht erreicht.

Im Zuge dieses explosionsartigen Wachstums der Branche, besonders sichtbar seit 2007 (Jiandan Xinli Academic Research Group, 2016, S. 3), ist der Markt immer unübersichtlicher geworden. Wohl um möglichem Missbrauch vorzubeugen, hat das Gesundheitsministerium Anfang 2018 eine elementare und wenig praxisorientierte

1 Der besseren Lesbarkeit wegen habe ich abwechselnd maskuline und feminine Endungen verwendet. Wenn nicht explizit angegeben, sind damit automatisch beide Geschlechter gemeint.

2 Diese Schätzung basiert auf der von Phillips und Kollegen (2009) angegebenen Ein-Monats-Prävalenzrate für die erwachsene Bevölkerung in der Volksrepublik von $17,5 \%$. 
Form der Zertifizierung bis auf Weiteres ausgesetzt, um neue, einheitlichere Standards festzulegen und den Berufszugang mehr zu ordnen. Offensichtlich ist jedoch, dass es sich bei den angebotenen Psychotherapierichtungen, von wenigen Ausnahmen abgesehen (z. B. Moritatherapie, Kognitive Taoistische Therapie etc.), um solche westlichen Ursprungs handelt. Neben klassischer und moderner Psychoanalyse, Kognitiver Verhaltenstherapie und Klientenzentrierter Therapie finden sich alle möglichen Verfahren wie Systemische Familientherapie (z.B. Virginia Satirs Modell), Lösungsorientierte Kurztherapie, Sandspieltherapie, Akzeptanz und Commitmenttherapie, Gestalttherapie, Rational-Emotive Therapie, aber auch Programme zur Supervision und Employee Assistance Programs> leicht in der therapeutischen Landschaft wieder. Dabei sind es vor allem drei Gruppen, die diese Beratung praktisch ausüben und weiterentwickeln: Akademiker in tertiären Bildungseinrichtungen inklusive der dortigen Psychotherapeutischen Beratungsstellen, Ärztinnen in Psychiatrischen Kliniken oder Abteilungen von grösseren Kliniken sowie private Unternehmerinnen, entweder selbstständig («Ich-AGs», auch oft im Online-Format) oder im Angestelltenverhältnis für Psychotherapiefirmen auf dem freien Markt.

\section{Was hat sich durchgesetzt?}

Da sehr viele Berater und Therapeutinnen eklektisch arbeiten und keine systematische langjährige Ausbildung genossen haben, ist es nicht leicht, verlässliche Aussagen darüber zu treffen, welche Verfahren sich nun «durchgesetzt` hätten und damit vielleicht «natürlicherweise` besser zur chinesischen Kultur 〈passen〉 könnten als andere. Vorhandene Zahlen und Expertenmeinungen gehen weit auseinander. ${ }^{3}$ Bei der Heterogenität des Landes und der unstrukturierten Situation für Therapeutinnen ist das nicht verwunderlich. Sicher ist jedoch, dass es einen starken Sog für sämtliche Psychotherapieformen gibt und immer mehr Verfahren mit grossem Lerneifer aufgenommen werden (vgl. Hillers-Chen, i. D., für eine ausführliche Diskussion). Ebenso sicher ist, dass alle Teilnehmenden - egal welchen kulturellen Hintergrundes - glauben, es müsse sich etwas ‘adaptieren', um China gerecht zu

3 Zum Beispiel fragt Zhang (2014) nach ihrer ausführlichen Feldforschung für die Gruppe der selbstständig arbeitenden Beraterinnen in Kunming: «Meine Forschung zeigt, dass, obwohl eine ganze Reihe von psychologischen Theorien und Behandlungsmodellen in China eingeführt worden sind, drei Ansätze an Beliebtheit gewonnen haben und verschiedenen Adaptionen unterzogen werden: Systematische Familientherapie, Kognitive Verhaltenstherapie (KVT), und Sandspieltherapie. Während Freud'sche Theorien und klassische Psychoanalyse als grundlegend für zeitgenössische Psychologie und Beratung angesehen werden, werden sie für gewöhnlich nicht bei der eigentlichen Beratung angewendet oder von Therapeutinnen als ihre Fachgebiete beworben. Warum das?» (S. 292, Übers. d. A.). Im Gegensatz dazu kommt Shi (2014) zu einer ganz anderen Einschätzung, nämlich dass «es bereits klar ist, dass die Psychoanalyse ein Mainstreamansatz in China geworden ist» (S. 157). Diese Beispiele veranschaulichen, wie heterogen die Ideen innerhalb des Gesamtfeldes sind. werden. Interessanterweise habe ich selten von der Frage gehört, was sich adaptieren müsse, um eine Therapieform zum Beispiel in der Schweiz anzuwenden, in den USA oder Südamerika. Die «westliche Verwandtschaft und damit Übertragbarkeit steht offensichtlich ausser Frage. Zuletzt ist auffällig, dass Vertreter eines bestimmten Verfahrens jeweils angeben, ihr Verfahren würde mit den entsprechenden Adaptionen sehr gut zu China passen. Folgende Aussagen mögen dies beispielhaft verdeutlichen:

«Diese Literaturübersicht zeigt, dass die Lösungsorientierte Kurztherapie (SFBT) erfolgreich in chinesischen Populationen angewendet werden kann, und das in vielen verschiedenen Praxisfeldern. [...] die praktische, an Stärken orientierte und optimistische Art, mit der SFBT an Probleme herangeht, funktioniert gut mit Chinesen und hilft diesen auch dabei, ihren Respekt gegenüber den Eltern und ihre Würde beizubehalten während sie ihren persönlichen Themen im Bereich psychischer Gesundheit entgegentreten» (Liu et al., 2015, S. 88, Übers. d. A.).

"Westliche Systemische Familientherapie bietet eine gute Passung für die Behandlung von einigen Problemen im chinesischen Kontext, vorausgesetzt, dass sie die Familie und die Verbundenheit zwischen den Generationen betont» (Miller \& Fang, 2012, S. 179, Übers. d. A.).

«In einer Zeit, in der sich jeder sorgt, zurückzufallen und im Ansturm verlassen zu werden, hat [Psycho-] Analyse das Potenzial, uns dabei zu helfen, uns nicht zu verlieren» (Xu et al., 2011, S. 205, Übers. d.A.).

«Die KVT [Kognitive Verhaltenstherapie] bietet beides, sowohl Möglichkeiten als auch Herausforderungen, wenn sie im Hinblick auf den chinesischen kulturellen Kontext adaptiert wird. Ermutigend ist, dass die meisten ihrer offenkundigen Herausforderungen mehr mit den praktischen Eigenschaften zu tun haben als mit den fundamentalen Prinzipien des therapeutischen Modells. Daher scheint es, dass die KVT modifiziert werden kann, um die Bedürfnisse und Präferenzen von chinesischen Klientinnen zu befriedigen, ohne dass die sie definierenden Charakteristika beeinträchtigt werden» (Guo \& Hanley, 2015, S. 61, Übers. d. A.).

Was die Adaptationsfähigkeit von Psychotherapie und Beratung betrifft ist ein reichhaltiges Spektrum an Sichtweisen vertreten: Einige Fachvertreter stellen - zumindest ansatzweise - das Konzept von «Psychotherapie» an sich in Frage, weil es einfach gar nicht zur chinesischen Kultur passe, während andere Fachvertreter glauben, all die Diskussionen um kulturelle Passung seien übertrieben, da Menschen nun einmal nach ähnlichen Prinzipien funktionierten und Anpassungen eher vernachlässigbar seien. Beide Positionen werden meiner Beobachtung nach eher mündlich vorgetragen, wie zum Beispiel von Prof. Hong $\mathrm{Fu}$ (männlich) und Prof. Xudong Zhao (männlich) auf 
dem Forum für Psychische Gesundheit und Beratung unter Berücksichtigung der Kultur der Chinesen ${ }^{4}$ (Zhao, 2018). Prof. Fu bemerkte dabei unter anderem, dass ihm Psychotherapie wie eine Weiterführung der christlichen Tradition der Beichte erscheine, die dem chinesischen Kulturraum völlig unbekannt sei: Probleme würden traditionell eher innerhalb der Familie gehalten. Prof. Zhao erinnerte jedoch daran, dass er als Teilnehmer der ersten Kohorte der Therapieausbildung, durchgeführt von der Deutsch-Chinesischen Vereinigung für Psychotherapie, nie auf grössere Unterschiede gestossen sei und man die Kultur bei der Frage nach geeigneten Behandlungsmethoden nicht überbewerten sollte: Inhalte seien vielleicht unterschiedlich, die Mechanismen gleich.

In einer Literaturrecherche zum Thema Beratung, Therapie, Kultur und Indigenisierung in Chinas grösster nationalen Datenbank CNKI (Chinese National Knowledge Infrastructure) fand ich ausschliesslich Artikel, in denen eine Adaptation oder Indigenisierung von Psychotherapie und Beratung gefordert wird. Das ähnelt den Diskussionen innerhalb der Psychologie, die sich mit der Schnittstelle zu Kultur befassen (vgl. z. B. Shweder, 2000; Triandis, 2000; Yang, 2000; aber auch das komplette Sonderheft im Asian Journal of Social Psychology 3/2000, in dem die Hauptvertreter der ‘grossen Drei - Indigene, Kulturelle und Kulturvergleichende Psychologie - sehr unterschiedliche Auffassungen davon, wie Psychologie vor unterschiedlichen kulturellen Hintergründen gedacht werden könne). Auch hier spielen sich die Grabenkämpfe zwischen einem moderaten Relativismus und einem moderaten Universalismus menschlichen Verhaltens ab. Und während der Umgang mit Kultur einerseits immer vielfältiger wird und immer mehr die Hybridität von Kulturen in den Vordergrund geschoben wird (vgl. Hermans \& Kempen, 1998; van Oudenhoven \& Ward, 2013; oder in Bezug auf China und zum Thema "Culture mixing: It's nature and psychological implications": Lonner, 2016; sowie die vielen Wortschöpfungen, die vormals postulierte Gegensätze versuchen aufzulösen: inter-, trans-, multi-, omni-, poly-, n-, oder komplexkulturell), hält das die weniger an kulturellen Eigenheiten orientierte Mainstream-Psychologie in China andererseits nicht davon ab, de facto weiterhin in neo-positivistischen Strukturen zu denken. Es wird so immer schwieriger, sich innerhalb dieses Raums zu verorten.

\section{Streitrosse und Streitgespräche: Weitong juela}

Wenn ich meine Gespräche mit Fachvertreterinnen all die Jahre Revue passieren lasse, dann fällt auf, wie oft meine Gegenüber mit dem Terminus «chinesische Charakteristika» arbeiten. Chinesische Besonderheiten sind vor allem überall da, wo Lücken auftreten oder etwas schwer erklärbar wird. Meist bedarf es dem Vernehmen nach keiner weiterführenden Erklärung, so offensichtlich scheint die Validität solcher Aussagen. Aber was sind denn eigentlich chinesische Charakteristika?

4 Im Sinne von huaren (wird im Folgenden noch erläutert).
Die meisten Abhandlungen zu diesem Thema bleiben auf einem sehr hohen Abstraktionsniveau. Gerne werden Dichotomien bemüht, die dann in unterschiedlichen Ausprägungen Westlern bzw. Chinesen zugeschrieben werden, um die Unterschiede deutlich zu machen. Die typischen Streitrosse, die dann ins Feld geführt werden sind zum Beispiel Individualismus vs. Kollektivismus, unabhängiges Selbst vs. interdependentes Selbst, Schuldkultur vs. Schamkultur, geringe Machtdistanz vs. grosse Machtdistanz, analytisches Denken vs. holistisches Denken, Wettbewerb und Leistung vs. interpersonelle Harmonie, Stolz vs. Bescheidenheit, Orientierung am Einzelnen vs. Familienorientierung - um nur einige zu nennen. Darauf wird dann auch im Hinblick auf Psychotherapeutische Beratung Bezug genommen. Yan (2008) schreibt zum Beispiel:

«Erstens, was die Motivation für Beratung betrifft, haben Menschen aus dem Westen einen aktiven Stil, Menschen aus dem Osten einen passiven. [...] Zweitens, was Beratungsergebnisse betrifft, schütten westliche Menschen ihr Herz aus, östliche Menschen sind problemlöseorientiert. [...] Drittens, was die beraterische und therapeutische Beziehung betrifft, haben Menschen aus dem Westen einen Stil wie Freunde, östliche Menschen einen wie Fremde» (S. 63).

Bereits hier wird deutlich, wie leicht Konzepte ausgedehnt werden (z.B. östlich statt Chinesisch) und wie widersprüchlich Erklärungen auf diesem Niveau sein können (z. B. wird Problemlöseorientierung im allgemeinen Diskurs eher Westlerinnen zugeschrieben, hier jedoch genau umgekehrt).

Wenn es dann in einem weiteren Schritt darum geht zu erklären, warum diese Unterschiede entstanden sind bzw. was die eigentliche Essenz Chinas sei, so dürfen in jedem Fachartikel die «drei Heiligen nicht fehlen: Konfuzianismus, Taoismus und Buddhismus. Diese drei Philosophien bzw. spirituellen Lehren sind es dann auch, die sogenannten indigenen Ansätzen ihren Geist einhauchen und damit eine kulturelle Passung verleihen, selbst wenn das Setting klassischer individueller Psychotherapie beibehalten wird, das heisst, eine Therapeutin und ein Klient 50 Minuten in einem Raum im Gespräch sind.

Neben solch notwendigen Vereinfachungen, um überhaupt von allgemeinen Charakteristika sprechen zu können, ist mein eigentliches Problem mit diesen Konzepten - nach all meinen Jahren der Suche -, dass sie nicht gut zu meinen Erfahrungen in meiner Lebensrealität passen wollen.

Die Menschen in der U-Bahn integrieren eine Fülle von Modestilen, ohne dabei einer bestimmten Konvention zu folgen. Meine Studierenden könnten analytisch denkender nicht sein. Rankings und Wettbewerb (umgangssprachlich «PK» genannt ${ }^{5}$ ) sind so in das Leben hineingewoben, dass

5 Entsprungen aus der Online-Gamer-Szene als Abkürzung für player killer, als Bezeichnung für einen Spieler, der absichtlich andere Spieler tötet. 
man denken könnte, es sei eine chinesische Erfindung und «Harmonie» eher der Name für Hochgeschwindigkeitszüge im Land als für die allgemeine Mentalität der Gesellschaft. Mein Kollege und etablierter Chinaforscher Xuewei Zhai (vgl. v. a. seine beiden Werke zu den Themen Gesicht und Guanxi: Zhai, 2011, 2013) beklagt sich oft bei mir, dass nahezu jeder Westler, der ihm begegne, darauf bestehe, in seinem Land gäbe es eigene Besonderheiten, die nicht mit «der Westlerin an sich zu fassen seien, findet es aber selbstverständlich, dass China nicht überall homogen sei.

Ebenso zugespitzt gefragt: Wie viel Taoismus/Buddhismus/Konfuzianismus ssteckt e eigentlich noch in den Menschen nach 68 Jahren sozialistischer Staatsideologie, Kulturrevolution und knapp 30 Jahren Öffnungspolitik? In der psychologischen und psychotherapeutischen Fachwelt als Erklärungstheorie für kulturelle Unterschiede kommt der «Sozialismus mit chinesischen Charakteristika» (Xi, 2017) kaum vor bzw. wird als «westliche> Theorie abgetan, wahrscheinlich weil es ein sehr sensitives und politisch aufgeladenes Thema im Inland ist.

Prof. Zhai sieht den Konfuzianismus aufgrund seiner jahrelangen Verankerung in den Beamtenprüfungen für die höchsten Staatsämter als wichtigste Einflusstheorie an, deren Überbleibsel lediglich indirekt in der derzeitigen Realität sichtbar seien bzw. neuerdings wiederentdeckt oder rekonstruiert würden (wie seit Neuestem ausdrücklich und offiziell vom derzeitigen Staatspräsidenten Xi Jinping). Dieser neue Konfuzianismus hat sich stark von seinem Original entfernt (Solé-Farràs, 2014), wenn man ob der vielen Re-Interpretationen über die Zeit überhaupt von einem Original sprechen kann. Die Mehrzahl meiner Studierenden wird ihre Verwirrungen, Zukunftsentscheidungen oder psychischen Belastungen jedoch nicht nach konfuzianischer Doktrin mit ihren Vätern oder Grossvätern ausmachen, ähnlich wie in Deutschland immer weniger Menschen dafür zur Beichte gehen. Keine der Teilnehmenden meines aktuellen Masterstudiengangkurses haben je ein Auge auf das Yi Qing (Buch der Wandlungen) geworfen. Und mein aktueller Undergraduatekurs versucht zwar pflichtbewusst, die chinesische Perspektive auf westliche Störungsbilder gemäss DSM-5 zu erarbeiten, kann aber mit den Erklärungsansätzen wie der Lehre der fünf Elemente nichts anfangen. Die Vortragenden sind meist sehr beschämt, dass es da «nichts Wirkliches» gäbe ausser Anekdoten. Manche von ihnen verstehen auch meine Fragestellung nicht: Welche chinesische Perspektive - Depression ist doch Depression, und Psychotherapie die Art, wie man sie behandelt, oder? Das ist es wohl, was Nikola Spakowski, Professorin für Sinologie an der Universität Freiburg, meinte, als sie in einem Gespräch mit mir von der Dekonstruktion Chinas sprach. Je näher man hinsieht, desto weniger überzeugend wirken die üblichen Abstraktheiten.

Hinzu kommt speziell für Psychologinnen, was Ratner und Hui (2003, S. 74) bereits früh im Hinblick auf kulturvergleichende Psychologie im Allgemeinen und für das Beispiel China im Besonderen beklagen, dass diese nämlich schlecht über Soziologie und Geschichte informiert sind und daher kulturelle Faktoren und ihre psychologischen Effekte missverstehen, von anderen methodischen Schwierigkeiten für die empirische Forschung einmal ganz abgesehen (eine ausführliche Betrachtung dieses Themas würde den Rahmen dieses Beitrags überschreiten). Das Phänomen ist dabei beidseitig: Beteiligt sind sogenannte westliche Psychotherapeuten mit dem, was sie über ihr eigenes Land, China und die Welt wissen bzw. konstruiert haben, sowie die chinesischen Therapeutinnen mit dem, was sie über ihr eigenes Land, «den Westen` und die Welt wissen bzw. konstruiert haben - und ihr via Übersetzung ${ }^{6}$ hergestelltes Verständnis miteinander. Je nach Verständnisgrad, Wissensstand und Integrationsniveau werden dann bestimmte Phänomene nicht nur als typisch empfunden, sondern auch mit den jeweils bekannten Elementen des Diskurses in Verbindung gebracht. Und da die grossen Dichotomien und die drei grossen Theorien sehr populär und am zugänglichsten sind, sind solche abstrakten Erklärungen recht schnell bei der Hand, lassen mich jedoch meist unbefriedigt zurück.

Von den von mir für diesen Beitrag recherchierten 32 Artikeln in sogenannten chinesischen Kernjournalen (hexin qikan) zum Thema Psychotherapie und Beratung und chinesischer Kultur in China basieren alle auf solchen vereinfachenden zusammenfassenden Überlegungen. Kein einziger Artikel stellt eine empirische Analyse zu den beschworenen Charakteristika an oder diskutiert wenigstens die Frage zur Kultur oder wie der Begriff verwendet wird. Dabei könnte es eigentlich leicht Anknüpfungspunkte für eine vertiefende Diskussion geben. Hong und Kollegen (2010, S. 19) geben im Oxford Handbook of Chinese Psychology gleich acht verschiedene Interpretationen zur möglichen Bedeutung von "Chinesischer Psychologie» ${ }^{7}$ an und weisen ausserdem auf den Unterschied von zhongguoren und huaren hin. Beides sind chinesische Ausdrücke für "Chinesen», ersterer bezieht sich auf die Bewohner des politischen Gebildes des heutigen Landes China (das auch nicht eindeutig ist), letzterer auf eine vage kulturelle Gemeinschaft von In- und Auslandschinesinnen vereint in einem chinesischen Ursprung (meist im Sinne von Mutterland oder sogar Blutsverwandtschaft). Am Ende bleiben auch hier die Interpretationen vielfältig und das Konzept vage. Je nach Interpretation ist eine Erfahrung damit typisch chinesisch oder eben nicht oder, anders ausgedrückt, beides gleichzeitig.

Dieses Thema ist für mich daher mittlerweile weitong juela geworden, also «einheitlich im Geschmack als wäre das, was ich kaue, Wachs» wie «die» Chinesen sagen.

6 Diese Thema wäre an sich schon einen eigenen Artikel wert. Speziell für chinesische Übersetzungen sei auf die Arbeiten von Sonya Pritzker verwiesen, z. B. Pritzker \& Hui (2014), oder seit Neuestem das Routledge Handbook of Chinese translation (Shei \& Gao, 2018).

7 (1) Psychologie über China oder die Chinesische Kultur, (2) den Chinesen oder China gehörend, (3) in China entstanden, (4) praktiziert, kreiert oder von Chinesen konsumiert, (5) in China von Chinesen praktiziert, (6) mithilfe von Chinesischen Stimuli, Forschungsmaterialien und Forschungsprobanden konstruiert, (7) aus China exportiert, (8) hergestellt für den Nutzen und Konsum in China oder von Chinesen. 
Natürlich nehme ich Unterschiede wahr, die ich als Kulturunterschiede interpretiere, diese sind für mich jedoch nicht leicht analytisch fassbar, sie rinnen durch meine Finger, sobald ich sie festlegen möchte. Gleichzeitig spüre ich auch immer weniger das Bedürfnis, sie ausdrücken zu wollen, und finde mehr Gefallen an metaphorischen Umschreibungen. Die Diskussion um die chinesischen Charakteristika ist wie ein Spiel, das alle Beteiligten brauchen, um sich immer wieder zu vergewissern, wie sie nicht sind oder doch sind, als Versicherung ihrer Einmaligkeit und Bedeutsamkeit. Viel zu selten wird eigentlich die Frage gestellt, wer die Frage «Was sind die chinesischen Charakteristika?» stellt und welche Machtposition diese sprechende/fragende Person innehat.

Psychotherapie ist neben einer Einnahmequelle für westliche und chinesische Institute längst auch eine Form der Machtausübung geworden, die gezielt von der Regierung für ihre Zwecke eingesetzt wird (s. hierzu die sehr detaillierte Analyse der Anthropologin Jie Yang, 2018, die dafür den Ausdruck «therapeutisches Regieren»/«therapeutic governance» benutzt; in die gleiche Richtung gingen auch einige mündlich vorgetragene Warnungen an Therapeutinnen auf dem o.g. Forum). So wird beispielsweise immer mehr deutlich, dass die psychischen Folgen der rapiden Umwälzungen nicht mehr allein vom Staat zu bewältigen sind. Im Zuge dieser Verlagerung der Verantwortung für die Bewältigung von gesellschaftlichen Problemen auf das Individuum wird in Form von Psychotherapie gezielt eine neue Form der Selbstfürsorge für die wachsende neue Mittelschicht geschaffen. Inwiefern ein solch gelenkter Einsatz mit den Grundsätzen einiger Therapieverfahren vereinbar ist, ist sicher eine weiterführende Diskussion wert. Gegenwärtig treten solche Fragen jedoch eher in den Hintergrund, während die Befriedigung der grossen Nachfrage vorrangig bleibt.

Natürlich kann es niemand leisten, gleichzeitig Psychologe, Geschichtswissenschaftlerin, Soziologe, Philosophin, Politikwissenschaftler und Linguistin in eigener Sache zu sein, aber ein wenig mehr Dialog mit der eigenen und der jeweils anderen Geschichte würde die Komplexitäten wieder mehr in den Vordergrund rücken. Gleichzeitig wäre es sehr spannend, expliziter an den Veränderungsprozessen eines eingewanderten Verfahrens teilhaben zu können, denn, wie ein chinesischer Kollege es ausdrückte: Wir adaptieren überall, nur reden wir nicht so viel darüber.

\section{Was tun mit der Polyphonie der Stimmen? Präferenzen und Ausblick}

Bei den riesigen Umwälzungen der letzten Jahre sind die Menschen in meinem direkten Umfeld selbst immer unsicherer geworden, was ihre Identität im Kern eigentlich ausmacht. China ist eine komplexe, widersprüchliche Mischung aus Alt und Neu, Tradition und (Supra-)Moderne, Verletztheit und Stärke. Oder wie Yu Hua es in seinem autobiografischen Roman China in zehn Wörtern (2011) zusammenfasst: «Das China von heute ist ein Land der grossen Unterschiede. Es ist [...], als ob man in einem eigenartigen Theater sässe, wo eine Komödie auf der einen Seite der Bühne aufgeführt würde und eine Tragödie auf der anderen» (S. 117, Übers. d. A.). Und auf dieser Bühne müssen sich dann auch die ‘westlichen` Therapieansätze bewegen, um bei der Metapher zu bleiben.

Die eingewanderten Theorien sind für mich trotz der vielen Beteuerungen, dass sie es eigentlich nicht sind, bereits ein Teil Chinas und somit in dem Sinne "chinesisch", zumal so viele Menschen sich auf sie stürzen. Wie oben bereits ausgeführt, ist für mich vor diesem Hintergrund eine abstrakte Top-down-Analyse weniger interessant als eine gemeinsame Betrachtung von Schwierigkeiten, die bei der Durchführung von westlichen Therapieansätzen in China entstehen. Ob diese dann auf chinesische Besonderheiten hinweisen und, eigentlich viel interessanter, ob für diese Schwierigkeiten passende Lösungsoptionen kompatibel mit einem bestimmten Therapieansatz gefunden werden können, kann nur im Dialog mit erfahrenen Vertreterinnen der jeweiligen Verfahren aus beiden Kulturen entstehen.

So empfanden beispielsweise die chinesischen Teilnehmenden unseres zweijährigen Weiterbildungsprogramms in Gestalttherapie durchgeführt vom Institut für Integrative Gestalttherapie Würzburg (IGW) als schwierig, ohne Manual zu arbeiten. Sie wünschten sich eine Struktur, die genau anzeigen könnte, was sie im Therapieverlauf wie und wann genau tun sollten (Hillers-Chen, i.D.). Weist dies nun auf eine (chinesische Charakteristik hin? Im Dialog mit den ausländischen Trainerinnern und Trainern des IGW stellte sich heraus, dass dieses Anliegen auch ein häufig berichtetes Anliegen der Auszubildenden in deutschsprachigen Ländern ist, also nichts Ungewöhnliches darstellt, sondern eher der auch «im Westen` wohl nicht selbstverständlichen Denkart der Gestalttherapie geschuldet ist. Eine chinesische Teilnehmerin schlug sogar eine Art Handbuch mit Ablaufplänen und Entscheidungsbäumen vor. Könnte hieraus ein Wunsch nach Kontrolle abgeleitet werden? Oder ein grösserer Wunsch nach Kontrolle als im Westen`? Oder einfach nur eine Vorerfahrung der Teilnehmerin mit Kognitiver Verhaltenstherapie und klassifikatorischer Diagnostik à la SKID-Interview? Und wäre Ersteres dann eine chinesische Charakteristik und Letzteres lediglich ein Indiz, wie tief westliche Ideen in Teilen Chinas schon verankert sind?

In dieser Polyphonie der möglichen Interpretationen finde ich es persönlich spannend, nach sogenannten «gegenseitigen Huch!»-Reaktionen (engl.: mutual yuck! response; Shweder, 2000, S. 216f.) Ausschau zu halten, also nach Momenten der eher negativ konnotierten Überraschung, in denen spontan das Gefühl auftritt, dass eine Idee, Intervention, Sicht der Dinge einfach nicht passt. Treten diese regelmässig und bei vielen Therapeutinnen und Beratern in ihrem Handlungsfeld (während der Ausbildung und der Arbeit mit Klienten) auf, könnten solche Momente die Grundsteine für einen Bottom-up-Ansatz zur Erforschung von Besonderheiten sein, die dann im gegenseitigen Dialog mit Vertretern aus beiden Kulturbereichen explizit diskutiert und weiterentwickelt werden könnten. Ich kenne viele Anekdoten von 
Therapeutinnen sowohl in China als auch in Deutschland (ähnlich wie z.B. in Schlösser, 2009), was ihnen in der Arbeit mit dem jeweils anderen aufgefallen ist. Wie weit die Bewertungen dann allerdings auseinander liegen können, vor allem wenn sie nicht im Dialog expliziert werden, zeigt eine selten veröffentlichte Debatte um die Situation von psychotherapeutischer Beratung von Universitätsstudierenden (Portnoy, 2013 vs. Zhang et al., 2016). Zwar gibt es immer mehr Versuche, die Passung von einer Therapieform empirisch zu bestimmen (vgl. z. B. Liu et al., 2013, erneut mit positiven Befunden für eine mögliche Anwendbarkeit des Mailänder Modells für Familientherapie, oder Xu \& Tracey, 2016, die in einem Vergleich verschiedener Ansätze einen Passungsvorteil für humanistisch-erfahrungsbasierte Therapieformen ausmachen), aber eine systematische Untersuchung solcher Kleinstmomente, die einen Ansatz gemäss des Gelernten umzusetzen versuchen, ist mir im Feld beobachtet kaum bekannt.

Zwei Ausnahmen stellen erstens eine Analyse von Fokusgruppen chinesischer Auszubildenden in Systemischer Familientherapie zu genau diesem Thema $(N=16$; Queck \& Chen, 2017) und zweitens eine mehrjährige Feldbeobachtung von selbstständig arbeitenden Psychotherapeutinnen $(N=32)$ in der Stadt Kunming (Zhang, 2014) dar. Beide Abhandlungen geben sehr konkrete Beispiele, die die Breite und Komplexität des Kulturierungsprozesses verdeutlichen, und nennen explizit die Rolle der Interpretierenden bei der Datenanalyse. Queck und Chen sind beispielsweise Auslandschinesinnen, also buaren, aus Singapur und Taiwan nun beheimatet in den USA ( «Asian American»), die eine Analyse auf dem chinesischen Festland durchführen. Statt der üblichen Dichotomien finden sie viele Hinweise auf die Rolle der Finanzierbarkeit, ${ }^{8}$ auf den Zeitdruck, auf die Einstellungen und Selbstauffassungen unterschiedlicher Generationen (pre 1960er, 1970er und 1980er), darauf, wie Familie im Moment gelebt wird/ werden muss, auf die «neblige und komplizierte» (Zhang, 2014, S. 300; Übers.d.A.) Rolle der therapeutischen Beziehung, auf die Wahrnehmung der Therapeutinnen als Autorität, die direktiv Änderungsvorschläge machen soll, ${ }^{9}$ auf Denkarbeit in sozialistischer Tradition, auf die Rolle von chinesischen Metaphern, auf das Stigma von Psychotherapie besonders in ländlichen Regionen (während die meisten Psychotherapeuten ein Grossstadtleben gewohnt sind) etc. All dies sind wichtige Anhaltspunkte für einen weiterführenden Dialog, wie Therapieformen mit solchen Phänomenen umgehen könnten und wollten. Solche expliziten Analysen werden dann natürlich wiede-

8 Ein durchschnittliches Honorar von 500 RMB pro Therapiesitzung ist bei einem für die Mehrheit in Kunming durchschnittlichen Monatseinkommen von ca. 2500 RMB ein enormer Betrag (vgl. Zhang, 2014, S. 294f.).

9 "Am Ende der ersten Sitzung wollen sie [die Klientinnen] schon wissen, welche spezifischen Massnahmen sie ergreifen sollen. Sie glauben, es ist meine Verantwortung, ihnen Wissen und Rat zu geben, wofür sie schliesslich gerade bezahlt haben. Sonst glauben sie, dass ich ihnen etwas schulde oder einfach eine Betrügerin bin» (zit.n. Zhang, 2014, S. 300, Übers. d. A.). rum das Selbstverständnis der Theorien an sich verändern. Kulturierung ist damit immer ein weiterführender reziproker Prozess des Sich-miteinander-aneinander-Reibens und Sich-miteinander-Veränderns, also gleichzeitig immer auch Rekulturierung des Originals. Egal ob sich Therapie als komplexe Sozialform nun in China durchsetzen und verbreiten wird oder nicht, dieses Eintreten in den Prozess des Sich-Reibens im Konkreten ist an sich schon wertvoll für beide Seiten. Die Frage nach den chinesischen Charakteristika wird dabei nebensächlich.

\section{Literatur}

Guo, F. \& Hanley, T. (2015). Adapting cognitive behavioral therapy to meet the needs of Chinese clients: Opportunities and challenges [Die Adaptation von kognitiver Verhaltenstherapie, um den Bedürfnissen von Chinesischen Klienten gerecht zu werden: Gelegenheiten und Herausforderungen]. Psych Journal, 4(2), 5565. https://doi.org/10.1002/pchj.75

Hermans, H. J. M. \& Kempen, H. J. G. (1998). Moving cultures. The perilous problems of cultural dichotomies in a globalizing society [Bewegliche Kulturen: Die gefährlichen Probleme von kulturellen Dichotomien in einer sich globalisierenden Gesellschaft]. American Psychologist, 53, 1111-1120. https://doi.org/10.1037/0003066X.53.10.1111

Hillers-Chen, A. (i.D.). Gestalttherapie in China - «Geht das?». In U. Grillmeier-Rehder \& G. Pernter (Hrsg.), Spiegelungen des Hier-und-Jetzt: Gestalttherapie im Wandel der Zeit. Einblicke in gestalttherapeutische Theorie und Praxis aus 40 Jahren IGW-Ausbildungserfahrung. Köln: EHP.

Hong, Y.-Y., Yang, Y. J. \& Chiu, C.-Y. (2010). What is Chinese about Chinese psychology? Who are the Chinese in Chinese psychology? [Was ist Chinesisch an der Chinesischen Psychologie? Wer sind die Chinesen in der chinesischen Psychologie?]. In M.H. Bond (Hrsg.), The Oxford Handbook of Chinese Psychology (S. 19-29). Oxford: Oxford University Press.

Jiandan Xinli Academic Research Group (Hrsg.). (2016). 2016 diaocha baogao [Untersuchungsbericht 2016]. https://jdxl-file.b0.upaiyun.com/uploads/report2016.pdf (22.05.2018).

Kim, J.S., Franklin, C., Zhang, Y.P., Liu, X. W., Qu, Y.Z. \& Chen, H. (2015). Solution-focused brief therapy in China: A meta-analysis. [Lösungsorientierte Kurztherapie in China: Eine Metaanalyse]. Journal of Ethnic \& Cultural Diversity in Social Work, 24, 187201. https://doi.org/10.1080/15313204.2014.991983.

Liu, L., Miller, J.K., Zhao, X.D., Ma, X.Q., Wang, J.K., Li, W.T. (2013). Systematic family psychotherapy in China. A qualitative analysis of therapy process [Systematische Familientherapie in China: Eine qualitative Analyse des Therapieprozesses]. Psychology and Psychotherapy: Theory, Research, and Practice, 86, 447465. https://doi.org/10.1111/j.2044-8341.2012.02075.x

Liu, X., Zhang, Y.P., Franklin, C., Qu, Y., Chen, H., \& Kim, J.S. (2015). The practice of solution-focused brief therapy in mainland China [Die Praxis von lösungsorientierter Kurztherapie auf dem Festland Chinas]. Health \& Social Work, 40(2), 84-90. https://doi. org/10.1093/hsw/hlv013

Lonner, W. J. (2016). Introduction to the special issue [Einführung in die Sonderausgabe]. Journal of Cross-Cultural Psychology, 47(10), 1255-1256. https://doi.org/10.1177/0022022116672586

Miller, J.K. \& Fang, X. Y. (2012). Marriage and family therapy in the People's Republic of China: Current issues and challenges [Eheund Familientherapie in der Volksrepublik China: Aktuelle Aspekte und Herausforderungen]. Journal of Family Psychotherapy, 23(3), 173-183. https://doi.org/10.1080/08975353.2012.705626

Phillips, M.R., Zhang, J., Shi, Q., Song, Z., Ding, Z., Pang, S., Li, X., Zhang, Y. \& Wang, Z. (2009). Prevalence, treatment, and as- 
sociated disability of mental disorders in four provinces in China during 2001-2005: An epidemiological survey [Prävalenz, Behandlung und damit verbundene Beeinträchtigungen von psychischen Störungen in vier Provinzen Chinas zwischen 2001-2005]. The Lancet, 373(9680), 2041-2053. https://doi.org/10.1016/S01406736(09)60660-7

Portnoy, R. N. (2013). A psychology Fulbright year in China [Ein Psychologie Fulbrightjahr in China]. Journal of College Student Psychotherapy, 27, 104-109. https://doi.org/10.1080/87568225.2013 .766098

Pritzker, S. \& Hui, K.-K. (2014). Introducing considerations in the translation of Chinese medicine [Einführende Betrachtungen zur Übersetzung von chinesischer Medizin]. Journal of Integrative Medicine, 12, 394-396. https://doi.org/10.1016/S20954964(14)60032-7

Queck, K. M.-T. \& Chen, H.-M. (2017). Family therapy in Chinese culture and context: Lessons from supervising therapists-in-training in China [Familientherapie in chinesischer Kultur und chinesischem Kontext: Einsichten aus der Supervision von Ausbildungskandidaten zum Therapeuten in China]. Contemporary Family Therapy, 39, 12-20. https://doi.org/10.1007/s10591-016-9401-y

Ratner, C. \& Hui, L.M. (2003). Theoretical and methodological problems in cross-cultural psychology [Theoretische und methodische Probleme in der kulturvergleichenden Psychologie]. Journal for the Theory of Social Behaviour, 33(1), 67-94.

Shei, C. \& Gao, Z.-M. (Hrsg.). (2018). The Routledge handbook of Chinese translation [Das Routledge Handbuch für Chinesische Übersetzung]. London: Routledge.

Shi, Q. (2014). The development of psychoanalysis in China [Die Entwicklung der Psychoanalyse in China]. In D. E. Scharff \& S. Varvin (Hrsg.), Psychoanalysis in China (S. 157-165). London, England: Karnac Books.

Schlösser, A.-M. (2009). Oedipus in China: Can we export psychoanalysis? [Ödipus in China: Können wir Psychoanalyse exportieren?] International Forum of Psychoanalysis, 18, 219-224. https:// doi.org/10.1080/08037060902978760

Shweder, R.A. (2000). The psychology of practice and the practice of the three psychologies [Die Psychologie der Praxis und die Praxis der drei Psychologien]. Asian Journal of Social Psychology, 3, $207-$ 222. https://doi.org/10.1111/1467-839X.00065

Solé-Farràs, J. (2014). New Confucianism in twenty-first century China [Der neue Konfuzianismus im China des 21. Jahrhunderts]. London: Routledge.

Sun, S. F. \& Bitter, J.R. (2012). From China to South Korea: Two perspectives on individual psychology in Asia [Von China nach Südkorea: Zwei Perspektiven auf Individualpsychologie in Asien]. The Journal of Individual Psychology, 68, 233-248.

Triandis, H.C. (2000). Dialectics between cultural and cross-cultural psychology [Die Dialektik zwischen kultureller und kulturübergreifender Psychologie]. Asian Journal of Social Psychology, 3, 185-195. https://doi.org/10.1111/1467-839X.00063

van Oudenhoven, J.P. \& Ward, C. (2013). Fading majority cultures: The implications of transnationalism and demographic changes for immigrant acculturation [Schwindende Mehrheitskulturen: Die Implikationen von Transnationalismus und demographischem Wandel für die Akkulturation von Immigranten]. Journal of Community and Applied Social Psychology, 23, 87-97. https://doi. org/10.1002/casp.2132

Xi, J.P. (2017). The governance of China [China regieren]. Beijing: Foreign Languages Press.

Xu, H. \& Tracey, T.J.G. (2016). Cultural congruence with psychotherapy efficacy: A network meta-analytic examination in China [Kulturelle Kongruenz bei Psychotherapiewirksamkeit: Eine meta-analytische Netzwerkanalyse in China]. Journal of Counseling Psychology, 63(3), 359-365. https://doi.org/10.1037/cou0000145

Xu, Y., Qiu, J. Y., Chen, J. \& Xiao, Z.P. (2011). The development of psychoanalytic psychotherapy in Shanghai Mental Health Center [Die Entwicklung psychoanalytischer Psychotherapie am Shang- haier Zentrum für psychische Gesundheit]. International Journal of Applied Psychoanalytic Studies, 8, 196-206. https://doi. org/10.1002/aps.302

Yan, J. (2008). Wenhua xinlixue shiye xia de xinli zixun yu zhiliao de bentuhua. [Die Indigenisierung von psychologischer Beratung und Therapie aus der Perspektive der Kulturpsychologie]. Jiaoyu Lilun Yu Shixian [Theory and Practice of Education], 9, 62-64.

Yang, J. (2018). Mental health in China [Psychische Gesundheit in China]. Cambridge/UK: Polity Press.

Yang, K.-S. (2000). Monocultural and cross-cultural indigenous approaches: The royal road to the development of a balanced global psychology [Monokulturelle und kulturübergreifende indigene Ansätze: Der Königsweg zur Entwicklung einer ausgeglichenen globalen Psychologie]. Asian Journal of Social Psychology, 3, 241263. https://doi.org/10.1111/1467-839X.00067

Yu, H. (2011). China in ten words (übers. v. A.H. Barr) [China in zehn Wörtern]. New York: Anchor Books.

Zhai, X.W. (2011). Zhongguoren de guanxi yuanli. Shikong zhixu, shenghuo yunnian ziji liubian [Prinzipien der chinesischen Guanxi. Zeit-Raum-Rangordnung, Sehnsüchte des Lebens und deren fliessende Veränderungen]. Beijing: Peking University Press.

Zhai, X. W. (2013). Renqing, mianzi yu quanli de zai shengchan [Gefälligkeit, Gesicht und die Reproduktion der Macht]. Beijing: Peking University Press.

Zhang, L. (2014). Bentuhua: Culturing psychotherapy in postsocialist China [Bentuhua: Wie Psychotherapie im post-sozialistischen China kulturiert wird]. Culture and Medical Psychiatry, 38, 283305. https://doi.org/10.1007/s11013-014-9366-y

Zhang, Y., Yang, W.S., Liu, M.B. \& Wu, M. Y. (2016). Challenges and difficulties about college counseling in China: Response to Portnoy (2013) [Herausforderungen und Schwierigkeiten bei der College Beratung in China: Eine Erwiderung auf Portnoy (2013)]. Journal of College Student Psychotherapy, 30(1), 64-74. https:// doi.org/10.1080/87568225.2016.1105670

Zhao, X.D. (2014). Opportunities and challenges for promoting psychotherapy in contemporary China [Gelegenheiten und Herausforderungen bei der Verbreitung von Psychotherapie im zeitgenössischen China]. Shanghai Archives of Psychiatry, 26(3), 157-159. https://doi.org/10.3969/j.issn.1002-0829.2014.03.007

Zhao, X.D. (Moderator). (Mai 2018). Wenhua yu xinli zixun [Kultur und psychologische Beratung]. Panel auf dem Huaren wenhua xia de xinli jiankang yu zixun gaofeng luntan [Forum für Psychische Gesundheit und Beratung unter Berücksichtigung der Kultur der Chinesen]. Jiangsu sheng shehui xinlixue xuehui [Vereinigung für Sozialpsychologie der Provinz Jiangsu], Nanjing University, Nanjing.

\section{In search of Chinese characteristics. Western therapy approaches in the People's Republic of China}

Western-style psychotherapy has become a booming market in China. Based on the current state of psychotherapy in the People's Republic, it is almost impossible to tell what approaches would prevail or which of them are better or less suited for the country. Furthermore, the general discussion is based on abstract dichotomies as well as the three classics, Confucianism, Taoism, and Buddhism, which are increasingly struggling to reflect the complex and quickly changing China of today. The localisation of «Chinese characteristics» can quickly be reduced to absurdity. One solution is the bring the applied complexities and specific actions into the foreground to understand the reciprocal process of interpersonal friction not as cultivation effort of a form of treatment towards China, but as a mutual transformation process, i.e., the cultivation and re-cultivation of holistic therapy forms. The question regarding the Chinese characteristics becomes incidental. 
Key-Words: Psychotherapy, Chinese characteristics, Cultivation, Indigenisation, People's Republic of China

\section{Alla ricerca della caratteristiche cinesi. Approcci terapeutici occidentali nella Repubblica Popolare Cinese} La psicoterapia secondo il modello «occidentale» in Cina è diventata un mercato fiorente. Nell'attuale stato della psicoterapia nella Repubblica Popolare Cinese è difficile poter determinare quali approcci si sono affermati o si siano adattati in modo migliore o peggiore al paese, in particolare i tentativi di dibattito generale basati su dicotomie astratte e sui tre classici Confucianesimo, Taoismo e Buddismo possono rappresentare sempre più difficilmente il quadro attuale, complesso e dai rapidi cambiamenti, chiamato Cina. Una collocazione delle «caratteristiche cinesi» può essere condotta facilmente ad absurdum. Una via d'uscita consiste di riportare più sullo sfondo le complessità e la gestione concreta sul campo e di comprendere il processo reciproco di contatto non come tentativo di acculturamento di una forma di terapia in Cina, bensì come processo di cambiamento bilaterale, vale a dire un acculturamento o un riacculturamento di una forma di terapia per intero. L'interrogativo relativo alle caratteristiche cinesi diventa quindi irrilevante.
Parole chiave: Psicoterapia, caratteristiche cinesi, acculturamento, indigenizzazione, repubblica popolare Cinese

\section{Die Autorin}

Annette Hillers-Chen ist Associate Professor für Angewandte Psychologie und psychotherapeutische Beraterin am Zentrum für Gesundheitserziehung und -forschung an der Universität Nanjing. Sie arbeitet zum Themenbereich Akkulturation in China und hat eine China-Kooperation mit dem Institut für Integrative Gestalttherapie Würzburg seit 2009 mit aufgebaut. Ende 2018 wird sie nach Hangzhou an die Universität Zhejiang überwechseln.

\section{Kontakt}

Nanjing University

School for Social and Behavioral Sciences

Department of Psychology

Xianlin Campus

Heren Lou \#315

Postal code: 210046

annette.hillers@alumni.uni-heidelberg.de 